Abstract

\title{
Feasibility Study on S-Band Microwave Radiation and 3D Thermal Infrared Imaging Sensor-Aided Recognition of Polymer Materials from ELVs ${ }^{+}$
}

\author{
Jiu Huang * and Zhengfu Bian \\ School of Environment and Spatial Informatics, China University of Mining \& Technology, Xuzhou 221116, \\ China; zfbian@cumt.edu.cn \\ * Correspondence: jhuang@cumt.edu.cn \\ + Presented at the 5th International Symposium on Sensor Science (I3S 2017), Barcelona, Spain, \\ 27-29 September 2017.
}

Published: 15 December 2017

With the increasing worldwide consumption of vehicles, abandoned end-of-life vehicles (ELVs) have been rapidly increasing for the last two decades. Metallic material scraps are easily recycled and reused from crushed ELVs, the mixture residues of elastomers, plastics, printed circuit boards (PCBs), foam materials, and woody materials, etc.; scraps are classified as hazardous materials, in which elastomers and plastics constitute the vast majority. Their further treatment is strictly restricted and according to new legislation in the principle industrial countries, they are required to be materially recycled to achieve $85-95 \%$ in mass by 2020 . However, there are neither sufficient theories nor technologies for the identification and separation of elastomers and plastics from the mixture residues with high efficiency. To make matters worse, most applied vehicle elastomers were black or dyed a dark color which makes them unavailable for use in spectral sensors. In this research, we provide a novel method by using S-Band microwave radiation together with 3D thermal infrared sensors for recognition of elastomers from ELVs. In this study, an industrial microwave emitter array with $2.45 \mathrm{GHz}$ was utilized as a radiation source. More than four kinds of crushed ELV residue mixtures were tested. The mixture residues were designed to be convoyed through the microwave radiation with certain periods. According to the chemical structure and additive difference, the residue materials' sensitivities to the microwave radiation were also different and further led to variation of rising temperature. The variations of heating effect were obtained by a 3D-thermal infrared imaging sensor and the residue scraps of different materials were recognized for further sorting. The results show that, in the tested mixtures, elastomers heated up to more than $30{ }^{\circ} \mathrm{C}$ have the best recognition efficiency, and a minimum temperature variation of $5^{\circ} \mathrm{C}$ is needed to successfully fully distinguish different materials; the mixture residues heated up to less than $22{ }^{\circ} \mathrm{C}$ were not able to be recognized. The plastics had only very limited heating effects through microwave radiation. The sorting efficiency was independent of particle size but could be influenced by microwave power and radiation period. Generally, a mixture of more than $75 \%$ elastomers and plastics could be successfully recognized. Conversely, some of the residue materials could be more sensitive with a different frequency band of microwave radiations; therefore, in further experiments, other bands should be implemented for tests.

Conflicts of Interest: The authors declare no conflict of interest.

(C) 2017 by the authors. Licensee MDPI, Basel, Switzerland. This article is an open access article distributed under the terms and conditions of the Creative Commons Attribution (CC BY) license (http://creativecommons.org/licenses/by/4.0/). 